\title{
CONEXIONES ENTRE LAS CONCEPCIONES DE NUESTRA PERSISTENCIA DIACRÓNICA Y DE LA TEMPORALIDAD EN AXIOLOGÍA ${ }^{1}$
}

\author{
Oscar Horta \\ Fundación Española para la Ciencia y la Tecnología / Rutgers University \\ OHorta@dilemata.net
}

\begin{abstract}
Resumen
La relación entre el problema de la temporalidad del valor y el de nuestra persistencia diacrónica prácticamente no ha sido explorada hasta ahora. Sin embargo, el análisis de cada una de estas cuestiones puede arrojar luz sobre la otra de manera interesante. Este artículo argumentará que las conexiones entre la asimetría de nuestras actitudes hacia el futuro y el pasado, y la cuestión de nuestra persistencia diacrónica pueden defenderse sin incurrir en modo alguno a una petición de principio.
\end{abstract}

Palabras clave: neutralidad temporal del valor, prejuicio hacia el futuro, identidad, persistencia diacrónica, tridimensionalismo, tetradimensionalismo.

\section{Abstract}

The relation between the problem of the temporality of value and the problem of our diachronic persistence has virtually not been explored so far. However, the analysis of these questions can shed light on each other in an interesting way. This paper argues that an explanation of the connections between the asymmetry of our attitudes towards the future and the past and the question of our diachronic persistence is possible without begging the question in any way.

KEYWORDS: temporal neutrality of value, bias towards the future, identity, diachronic persistence, three-dimensionalism, four-dimensionalism.

\section{Introducción}

1 El modo en el que las distintas concepciones de la temporalidad del valor y las teorías de nuestra persistencia pueden esclarecerse mutuamente no ha sido 
hasta el momento mínimamente investigado. Pero un examen de los vínculos presentes entre ambos problemas puede resultar de utilidad para una mayor comprensión tanto de la una como de las otras. Ello es así, por una parte, debido a que la consideración de cuáles pueden ser las condiciones para nuestra persistencia diacrónica implica necesariamente mirar hacia el futuro y/o el pasado. De este modo, las diferencias en la manera de representarnos lo que ya nos ha sucedido y lo que nos sucederá podrán tener consecuencias en lo relativo a nuestras posibilidades de comprender tales condiciones. Por otra parte, precisamente del examen de la cuestión de nuestra persistencia diacrónica se puede derivar un argumento a favor de una de las concepciones de la temporalidad del valor (la de la neutralidad). Para mostrar de qué forma puede esto ser así procederé aquí, en primer lugar, presentando en qué consiste cada uno de los dos problemas en cuestión, el de nuestra persistencia diacrónica y el de la temporalidad del valor. A continuación, propondré qué clase de solución se deriva para cada uno de estos problemas del examen de distintas cuestiones implicadas en el análisis del otro. Como se indicará en la conclusión, ello puede defenderse sin incurrir en ninguna clase de petición de principio.

\section{Concepciones de las condiciones para nuestra persistencia diacrónica}

Abordemos en primer lugar la cuestión de nuestra persistencia diacrónica. Esta puede formularse de forma muy simple: ¿cómo puede suceder que un mismo ente exista en momentos distintos? Este problema va ligado, como se puede apreciar con facilidad, al de nuestra identidad. Y ambos temas implican, a su vez, la pregunta por la clase de cosa que somos. Para que podamos decir que un ente cualquiera $a$ es idéntico a $b$ debe suceder que ambos sean entes de un mismo tipo. Podemos decir que es así, evidentemente, porque si una entidad es idéntica a otra entonces ambas han de pertenecer, por necesidad, al mismo tipo. Pero el requisito, en realidad, va más allá de tal obviedad: sucede que las condiciones que $b$ ha de cumplir para ser $a$ dependen del tipo de entidades al que $a$ y $b$ pertenecen. Los requisitos que una manzana $p$ ha de cumplir para ser la misma manzana $q$ son distintos que los que una estrella $r$ ha de cumplir para ser la misma estrella $s$, aun cuando algunos puedan ser similares o coincidentes en ambos casos. Ello es así en función de la propia definición de cada tipo, de la cual será de donde se derivarán las condiciones de identidad de los entes que pertenezcan a este -lo que constituye la cláusula que Wiggins (2001; asimismo Locke 1968, III. iii.15). ha denominado "la dependencia de la identidad de la categoría tipificadora" (the sortal dependency of identity) $-^{2}$. Esta estipulación habrá de ser seguida tanto cuando lo que busquemos sea identificar sincrónicamente una cierta entidad como cuando lo que queramos sea reidentificarla diacrónicamente, esto es descubrir no ya

2 Conforme a la notación de Wiggins (2001) podemos expresar esta idea como sigue: $\mathrm{a}=\mathrm{b}$ (ヨf) $\left(\begin{array}{c}a=b \\ f\end{array}\right)$. 
si la entidad $a$ es idéntica a la entidad $b$, sino si la entidad $a$ existente en un momento $t_{1}$ es idéntica a la entidad $b$ existente en otro momento $t_{2}$. Podemos enunciar del modo siguiente cómo puede esto ser el caso:

Para todo $x$, y para dos momentos cualesquiera $t_{1}$ y $t_{2}, x$ existe en $t_{1}$ y $t_{2}$ si y solo si $x$ es una entidad de un cierto tipo $S$ y $x$ satisface cierta condición (o conjunto de condiciones) $C$ en $t_{1}$ y $t_{2}$.

La condición (o conjunto de condiciones) $C$ será, pues, aquella necesaria y suficiente para la persistencia a lo largo del tiempo de los entes del tipo $S$. En el caso de los entes de otros tipos $\left(S, S ", S^{\prime \prime}, \ldots\right)$, habrá que especificar otras condiciones para la persistencia $(C$ ' $C$ ",$C$ '”...), propias de su tipo. Con esto vemos que estamos ante dos problemas diferentes. El primero consiste en el esclarecimiento de la clase de entidades $S$ a la que $x$ pertenece. El segundo en la enunciación de las condiciones $C$ para la persistencia diacrónica de $x$. Ambos están, como es claro, ligados de un modo muy íntimo, pero constituyen cuestiones distintas. Más aun: puede de hecho ser el caso que descubramos cuáles son las condiciones $C$ para la persistencia de $x$ sin que hallemos a qué tipo de entidades $S$ pertenece, o que lleguemos a saber a qué tipo de entidades $S$ pertenece $x$ pero sin llegar a descubrir cuáles son las condiciones $C$ que determinan la persistencia de las entidades de este tipo. Por ejemplo, puedo quizás sostener que yo soy un ente que continuaré existiendo en la medida en que haya una determinada estructura física que albergue un flujo de conciencia, pero no saber propiamente si lo que soy es tal flujo de conciencia, sus contenidos, la citada estructura física en la medida en que se mantenga funcionando de tal modo que albergue tal flujo, etc. O puedo sostener que soy un flujo de conciencia, pero dudar de si seguiría existiendo si todas las partes de mi organismo fuesen paulatinamente sustituidas, o si la información de mis contenidos de conciencia fuese descargada en otro organismo que pudiese funcionar como el mío.

Pero la pregunta por la persistencia diacrónica implica una cuestión más básica, relativa no ya (o no solo) al tipo de cosas que son los entes que existen en ciertos momentos, sino al modo en que lo hacen: la de si las entidades subsisten ${ }^{3}$ o, por el contrario, perduran. En el primero de los casos, las condiciones para la persistencia hacen que un ente tridimensional vaya, por así decirlo, desplazándose temporalmente de un momento al otro: el ente $a$ en $t_{1}$ es idéntico al ente $b$ en $t_{2}$ (solo que en otro momento). En el segundo de los casos, lo que tales condiciones garantizan es que $a$ sea en $t_{1}$ una parte temporal del mismo ente tetradimensional que $b$ en $t_{2}$. La opción por una u otra alternativa se vuelve particularmente relevante de cara a la resolución de

3 Prácticamente no existe literatura en castellano sobre esta cuestión, la casi totalidad de lo que se ha escrito sobre esta ha sido en inglés. En la literatura anglosajona se utilizan los término 'to endure' y 'to perdure' para denotar, respectivamente, lo que aquí he optado por llamar 'subsistir' y 'perdurar'. 
ciertos casos particularmente problemáticos para la determinación de la identidad de una entidad, como son el de su fusión o su fisión ${ }^{4}$.

\section{Concepciones de la temporalidad del valor}

El problema más básico de la temporalidad del valor consiste en dar respuesta a la siguiente pregunta: ¿puede variar el valor de un beneficio o daño en función del momento $t$ en el que ocurre, si por lo demás permanece idéntico (e independientemente de cualquier relación que la ocurrencia de un beneficio o daño en $t$ pueda tener con algún otro beneficio o daño)? La respuesta que cabe dar por defecto a tal pregunta es negativa. En principio, mientras no haya otros factores que entren en juego, parece que hemos de asumir la que podemos denominar como tesis de la neutralidad temporal del valor (cf. Sidgwick 1996, p. 381). Esta es muy sencilla. Puede ser formulada del modo siguiente:

Dada una vida $v$ vivida por un individuo $i$ en la que $i$ recibe un cierto beneficio (o un daño) $b$ en un momento $t$, y dada otra vida $v$ 'que $i$ podría vivir que sería en todo igual a $v$ salvo en que $b$ afectaría a $i$ en otro momento $t_{2}$, el valor de $b$ para $i$ en $t_{l}$ es equivalente al valor de $b$ para $i$ en $t_{2}$, y, por lo tanto, el valor de $v$ para $i$ es idéntico al valor de $v$ ' para $i$.

Esta idea podría parecer, a primera vista, como intuitivamente clara. Lo que viene a expresar es, para decirlo de una forma menos comprometida ontológicamente, que el que un beneficio o un daño suceda en un momento $u$ otro no altera su magnitud. Sin embargo, el hecho es que, en cuanto examinamos las consecuencias que se derivan de ella, comprobamos que es rechazada en la práctica por la mayoría de nosotros. Normalmente se considera que los beneficios y daños futuros son importantes de un modo en el que no lo son aquellos que han sucedido en el pasado ${ }^{5}$. Se mantiene, pues,

$4 \quad$ Considérese el caso de un microorganismo que se fisiona en dos. Puede explicarse lo que sucede, sosteniendo que el organismo $o$ inicialmente presente desaparece y que dos nuevos organismos $o^{\prime}$ y $o^{\prime}$ ' hacen aparición, o que estos dos últimos son partes del primero (de modo que $o=o^{\prime}+o^{\prime \prime}$ ). O incluso que $o$ se multiplica en dos copias de sí mismo (lo que implica la dificultad de que acaban coexistiendo dos individuos idénticos, $o^{\prime}$ y $o$ ", que sin embargo difieren entre sí). Sin embargo, conforme a un modelo tetradimensional, cuando, cabe dar aun una solución más. Se puede sostener que los dos microorganismos resultantes $o$ ' y $o$ "existían ya con anterioridad a la fisión de manera superpuesta (no existiendo como tal $o$ más que como tal superposición). Esta solución, sin embargo, resulta imposible conforme a un modelo tridimensionalista.

5 Esto se pone de manifiesto en particular cuando se considera el que posiblemente sea el argumento más conocido que a lo largo de la historia ha apelado a la neutralidad temporal. Este no es otro que el que formulan Lucrecio (1976, 3, 830-842 y 972-976) y Cicerón (2005, I, 91) para invitarnos a no preocuparnos por la muerte, poniendo como ejemplo de 
una concepción del valor que da preferencia a lo que está aun por venir ${ }^{6}$. Esto se viene a corresponder con lo que Derek Parfit ha denominado, un "prejuicio hacia el futuro" -(bias towards the future- Parfit 1984, pp. 160-167 y 177-178). Consideramos que algo que nos suceda en el futuro es más importante para nosotros que lo sucedido en el pasado. Preferiríamos sufrir menos en el futuro, aunque ello supusiese que en el pasado habríamos sufrido más. Y, tal y como Ingmar Persson ha apuntado, dicha concepción viene ligada a una asimetría en lo que toca a nuestra capacidad de tener representaciones motivacionales de nuestras experiencias futuras y pasadas (Persson 2005 , p. 214). Ello es así porque somos capaces de representarnos lo que constituirá para nosotros el hecho de tener ciertas experiencias en el futuro de un modo particularmente motivador, que no está a nuestro alcance en el caso de experiencias pasadas. Sucede así incluso en el caso de que se trate de experiencias que no es posible que impidamos o promovamos (por ejemplo, si tenemos la certeza de que vamos a sufrir por la muerte de un ser querido que sabemos inminente). Esto es lo que hace que un sufrimiento futuro pueda, por ejemplo, suscitarnos miedo, sin que ello ocurra cuando recordamos un sufrimiento pasado similar ${ }^{7}$. He aquí, pues, el dilema al que nos enfrentamos: ¿hemos de seguir defendiendo la tesis de la neutralidad o renunciar a esta a favor de una primacía axiológica del futuro? ¿Cuenta realmente más un sufrimiento o disfrute por el mero hecho de que vaya a suceder en el futuro, en lugar de haber sucedido ya?

la que debería ser nuestra actitud ante ella nuestra relativa indiferencia hacia el hecho de no haber nacido antes. Este argumento puede ser rechazado por motivos diversos (fundamentalmente, por el hecho de que solo el futuro, y no el pasado, puede ser transformado). Pero la mayoría se opone a él por motivos distintos, más profundos, propiamente axiológicos, que tienen que ver con la preferencia por el futuro y el consiguiente rechazo de la neutralidad.

6 Otra posición común que se vendría a oponer a la neutralidad sería la constituida por la preferencia por lo último, que vendría a implicar que, ante secuencias de estados cuya suma es axiológicamente equivalente, se prefieran aquellas sucesiones en las que los mejores estados estuviesen situados al final, en lugar de al inicio. Preferimos, por ejemplo, una historia con un inicio triste y un final feliz a una que en un principio sea feliz pero que acabe mal, incluso aunque la duración e intensidad de la alegría y la infelicidad en cada uno de los dos casos sea la misma -como han apuntado Slote (1983, pp. 23-27) y Kamm (1993, pp. 41-42, 57-58 y 67-71). Esto es llamativo, debido a que esta preferencia se opone a la que también manifestamos por lo cercano, también opuesta a la neutralidad -lo cual han indicado Hume (1978, libro III, parte iii, sección 3) y Parfit (1984, pp. 158-165).

7 Tal capacidad tiene, pues, una orientación inversa a la de nuestra capacidad de conocer, temporalmente asimétrica en sentido contrario. Esto pone de manifiesto, así, una oposición (débil) en la dirección temporal de nuestras creencias y nuestros deseos. Si bien tanto nuestras creencias como nuestros deseos pueden dirigirse hacia el pasado como hacia el futuro, las primeras se hacen valer de modo más notable en el caso de lo que ya ha sucedido, mientras que los segundos se dirigen particularmente hacia lo que sucederá. 
4. Las condiciones de nuestra persistencia a la luz de nuestras actitudes hacia el tiempo

Una vez presentados los dos problemas, puede ya pasarse a considerar el modo en el que se pueden abrir puentes entre ambos. Comenzaré centrándome en el relativo a las condiciones para nuestra persistencia. Sostendré que el examen de los distintos factores que entran en juego en nuestra preferencia por el futuro puede ayudarnos a elucidar nuestros interrogantes respecto de este. Ello es así debido a que a menudo manifestamos una asimetría temporal a la hora de considerar qué clase de cosa somos y cuáles son las condiciones para nuestra persistencia. Comúnmente, cuando miramos hacia el pasado tendemos a identificarnos con los contenidos de conciencia conectados con nuestros estados psicológicos. Sin embargo, no sucede del mismo modo cuando oteamos el porvenir. Consideremos la posibilidad de sufrir amnesia total en el futuro. Hay quien mantiene que si ello sucede dejaremos de ser nosotros mismos (no ya en un sentido narrativo, sino metafísico, asumiendo que lo que nos distingue numéricamente desaparecerá si ello ocurre). Pero muchos considerarán que ello no será así: entenderán que en el caso de que suframos una amnesia total dejaremos de ser nosotros mismos en un sentido narrativo, pero continuaremos siendo numéricamente idénticos a la entidad que somos ahora. Esto se puede ver de forma más clara, considerando una versión, algo modificada, del famoso experimento mental lockeano del príncipe y el zapatero (Locke 1968, II.xxvii,.15). Supongamos que en el pasado hubiesen existido dos individuos: uno con un organismo diferente del mío y con contenidos de conciencia ligados a los míos, y otro con mi organismo y unos contenidos de conciencia totalmente inconexos con los míos. Muchos afirmarán que si alguno de estos dos individuos debería ser identificado conmigo, este habría de ser el primero. Sin embargo, si el problema es presentado con respecto al futuro, nuestra evaluación bien puede variar. Supongamos que dos individuos fuesen a existir en el futuro. Uno de ellos poseería mi organismo, pero no tendría ninguno de mis contenidos de conciencia actuales (ni tendría otros conectados mediante una cadena de continuos con los míos) ${ }^{8}$. El otro, poseería unos contenidos de conciencia conectados a los míos, pero tendría un organismo distinto del que ha estado tecleando para escribir estas líneas (en las versiones actuales de este ejemplo se presenta la situación considerándose que hemos sido objeto de un caso de traspaso mutuo de estados mentales o cerebrales sin que haya tenido lugar ningún intercambio de materia (Williams 1970, pp. 161-180). Muchos entenderemos que en un caso así yo continuaría estando en mi cuerpo independientemente de que sufriese un proceso de olvido y mis contenidos de conciencia dejasen de guardar relación con los que tengo en la actualidad.

8 Locke (1968, II, xxvii, 15). Véase también lo indicado por Parfit (1984, parte 3) o McMahan (2002). 
Estamos aquí, pues, ante dos intuiciones contrapuestas ${ }^{9}$, cada una de las cuales se hace valer según la situación que estemos considerando suceda en el pasado o en el futuro. En este sentido, podemos recordar lo apuntado en su momento en relación con la asimetría de nuestras representaciones motivacionales del futuro y el pasado. La constatación de nuestra mayor facilidad para tener representaciones motivacionales cuando miramos al futuro parece que constituye un motivo para confiar más en nuestras intuiciones hacia el futuro o, al menos, para preferir guiarnos por estas. El motivo radica en que nuestras representaciones de aquello que ya ha sucedido en el pasado serían más imperfectas, no nos presentarían las afecciones que podemos sufrir de un modo tan fiel. Y a la hora de plantearnos nuestras preocupaciones prudenciales en un experimento mental como el que aquí estamos considerando nuestra guía son nuestras representaciones motivacionales. Ello nos dota de un argumento a favor de una concepción que podemos llamar "sostenibilista" ${ }^{10}$. Esta rechazaría aquellas concepciones que, por ejemplo, afirman que la continuidad de nuestros contenidos de conciencia sería suficiente. En contraposición, situaría las condiciones de nuestra persistencia en la continuidad de un sustrato que proporcione un soporte a nuestra existencia a lo largo de la secuencia temporal en la que ésta se daría.

Es importante no perder de vista que este argumento no adopta como premisa nuestra preferencia por el futuro, sino únicamente la orientación hacia el futuro de nuestra capacidad de formar representaciones motivacionales.

\section{La temporalidad del valor a la luz del análisis de nuestra persistencia diacrónica}

Pasemos ahora al otro problema. Hemos visto que tenemos una preferencia hacia las experiencias que tendremos en el futuro. Ahora bien, si se nos pregunta por el porqué de tal preferencia, nuestras respuestas normalmente insisten en afirmar que nuestras experiencias futuras nos afectarán en algún momento, mientras las ya acaecidas han quedado atrás: no volveremos a pasar ya por ellas. Pero ello no es más que exponer de otro modo la misma idea que buscamos probar. Por otra parte, se puede considerar que el mero hecho de que tenemos tal preferencia es lo relevante, y que no hay motivo para cuestionarlo. Sin embargo, el hecho es que tenemos otras intuiciones que contradicen tal preferencia. La tesis de la neutralidad temporal es, por supuesto,

$9 \quad$ El propio Williams se vio perplejo ante este problema, y fue incapaz de optar por una $\mathrm{u}$ otra alternativa.

10 En realidad, esta solución sería también compatible con una posición que apuntase como condición para nuestra persistencia a la continuidad de la información relativa a nuestra constitución física. Conforme a esta posición, si existe un organismo que es cualitativa pero no numéricamente idéntico en un sentido físico al nuestro, nosotros seguimos existiendo. Esto no es así en el caso del sostenibilismo. 
una de ellas. Pero también lo es la idea, más general, de que en toda una serie de casos lo que preferimos acríticamente no es lo que preferiríamos si tuviésemos una información adecuada de los hechos y operásemos con esta de forma racional.

Pues bien, en realidad, sucede que desde un punto de vista puramente axiológico carecemos de razones para considerar prioritarios los beneficios o daños por venir. El propio análisis del valor, por sí mismo, puede variar en función de un factor temporal como la duración, pero no conforme a su localización. Cabe apuntar a este respecto la asimetría que se da con respecto al punto de vista que adoptamos desde dentro (una vez estamos localizados en algún punto temporal de nuestra vida) y el punto de vista externo, desde fuera, que se adopta con respecto a la hora de considerar el valor contenido en una vida cuando esta no es la que estamos viviendo nosotros en el momento en el que nos encontramos ${ }^{11}$. Supongamos que hemos de evaluar dos posibles situaciones, $s$ y $s^{\prime}$. Supongamos que ambas se dan antes y después de un cierto momento $t$. Antes de $t, s$ implica mucho sufrimiento para nosotros, y $s$ ' ninguno. Después de $t, s^{\prime}$ implica un poco de sufrimiento para nosotros, y $s$ ninguno. Desde un punto de vista externo, $s$ ' es claramente mejor, ceteris paribus. Desde un punto de vista interno, en $t$ la mayoría de nosotros preferiría, sin embargo, estar en $s$, a no ser que aceptemos la neutralidad. Axiológicamente, pues, la preferencia por el futuro resulta paradójica. No tenemos una justificación para ésta. De este modo, parece que el único modo de justificar nuestras actitudes preferentes hacia el futuro pasaría por mostrar que éstas pueden tener una base ontológica, puesto que la vía puramente axiológica parece cerrada.

En principio, las concepciones metafísicas de la temporalidad no aportan una solución favorable a la preferencia por el futuro: de hecho, la rebaten. Ello es así dado que ninguna de las teorías ontológicas del tiempo actualmente defendidas sostiene esta parcialidad hacia el futuro (como mucho, la sostienen hacia el pasado). Del presentismo $^{12}$ y eternalismo ${ }^{13}$ se vendría a derivar una posición temporalmente neutra, mientras que de la teoría del "bloque creciente" (growing block theory) ${ }^{14}$ se tendría que desprender una actitud contraria, a favor de lo que ya ha sucedido (al considerar el pasado y el presente existente, pero no el futuro $)^{15}$. Por supuesto, podemos considerar

11 Kamm (1993, p. 29). Considérese también a este respecto el argumento aportado por Parfit, (1984, pp. 181-184).

12 Véase, por ejemplo, la aportación de Chisholm (1990).

13 Véase Sider (2001). Pese a que el eternalismo no es en principio muy atractivo intuitivamente, se adecúa mejor a las implicaciones de la relatividad, en particular en lo que toca a la negación de la universalidad de la simultaneidad, que pone en apuros a todas aquellas teorías que ponen la frontera de lo existente en el presente, y muy particularmente al presentismo.

14 Esta posición es defendida por Tooley (1997).

15 No entraré aquí a valorar la repercusión que para la cuestión puede tener la opción por una teoría tensed o tenseless del tiempo. Parece que las conclusiones serían semejantes a las aquí planteadas. Más aun, como defendió en su momento Arthur Prior, la opción por una 
que esto es irrelevante, si consideramos que nuestra concepción axiológica del tiempo no tiene por qué venir condicionada por nuestra concepción ontológica de éste. Pero si es así, parece que el peso de la prueba recae en todo caso en las concepciones axiológicas no neutrales. Por supuesto, podríamos plantear la cuestión en otros términos e indicar los motivos (de tipo evolucionista, o aun termodinámicos) por los que tenemos actitudes temporales asimétricas. Pero esto únicamente explicaría por qué tenemos una cierta actitud, no nos daría una justificación de que tales actitudes sean las más apropiadas.

Nos queda comprobar, pues, qué respuesta cabría dar al problema desde el punto de vista de la identidad de los entes que persisten a lo largo del tiempo. Para ello no tomaré el sostenibilismo como premisa. Más aun, no me centraré, de hecho, en las concepciones de las condiciones para nuestra persistencia como tales, sino que adoptaré como herramienta unas teorías que las asumen, que las presuponen para tener sentido: las teorías del modo en que persistimos diacrónicamente. A la luz de éstas no solo no encontraremos un argumento a favor de la preferencia por el futuro, sino que veremos que la tesis de la neutralidad se ve reforzada. Lo que este argumento vendrá a indicar es que no hay ningún sentido en el que estemos más conectados con nuestro futuro que con nuestro pasado en aquello que debe ser prudencialmente relevante. Tal conexión es temporalmente simétrica. Si esto es así, adoptar una actitud prudencialmente distinta hacia el futuro y el pasado tiene que deberse a un error. La neutralidad temporal será la actitud que realmente proceda asumir.

Veamos, pues, cómo procede el argumento. Consideremos el caso de $b$, una entidad que existe en un cierto momento $t_{2}$. Conforme al tridimensionalismo, somos entidades idénticas que subsistimos diacrónicamente (van Inwagen 1990). Supongamos que es el caso que $b$ es diacrónicamente idéntica a dos entidades: $a$, que existe en el momento $t_{1} \mathrm{y} c$, que existe en el momento $t_{3}$, siendo $t_{1}$ anterior a $t_{2} \mathrm{y} t_{2}$ anterior a $t_{3}$. Se trata, pues, en todos los casos, de la misma entidad, localizada en distintos momentos. Ahora bien, si $a=b$, entonces $b=a$, y si $b=c$, entonces $c=b$, por supuesto. La identidad, pues, no es más manifiesta cuando miramos hacia el futuro que cuando lo hacemos hacia el pasado: $b$ no es más idéntico a $c$ que a $a$ por ocurrir $c$ en el futuro. Puede haber diferencias entre $a, b$ y $c$, pero éstas serán diferencias no relevantes de cara al mantenimiento de su identidad en función del tipo de cosas que son. De este modo, cabrá indicar que, si bien el tridimensionalismo podría ser en principio compatible con cualquier concepción de la temporalidad del valor, la respuesta que más bien parece darnos va bastante más en línea con la neutralidad.

Se podría aducir aquí que cuando hablamos de identidad para referirnos a lo que somos, lo hacemos solo en un sentido débil, puesto que solo podemos ser, verdaderamente, parcial o relativamente idénticos a un cierto ente que haya existido o existirá. Esta objeción, con todo, no apuntaría a lo que verdaderamente es el centro de la

concepción ontosemántica de la temporalidad tenseless vendría a poner en apuros a una posición contraria a la neutralidad. Véase Prior (1959). 
cuestión, debido a que la simetría se da igualmente en el caso de identidades relativas (Geach 1972, pp. 238-249) y, por otra parte, deja de lado que la identidad relevante es la relativa al sortal considerado.

¿Y qué sucede, a su vez, en el caso del tetradimensionalismo? Como es sabido, conforme a este, o bien somos entes que tenemos tanto partes espaciales como temporales (conforme a la "teoría del gusano" -worm theory-), o bien una de las partes de un ente tetradimensional (de acuerdo con la "teoría de las fases" -stage theory-) (Sider 2001). Parece, así, que esta teoría sería difícilmente compatible con una concepción no neutral de la temporalidad, particularmente en el caso de la teoría de las fases. Si estamos compuestos de partes temporales, no parece que haya en principio motivo alguno para que tengamos preferencia por algunas de tales partes antes que por otras en función de su posición en la cadena temporal: todas ellas están en posiciones simétricas ante y tras nosotros. Y menos aun tendremos razones para tener preferencia por alguna de las partes de un ente tridimensional por encima de otras si nosotros mismos no somos más que una parte más entre éstas. Al fin y al cabo, ninguna de tales partes será más identificable con nosotros mismos que la otra, si bien no, como en el caso del tridimensionalismo, porque todas ellas sean idénticas a nosotros, sino porque ninguna lo será. Así, los argumentos de carácter axiológico se ven respaldados por los derivados de la ontología de la temporalidad y la persistencia.

\section{Interconexión sin petición de principio}

A la vista de los argumentos arriba considerados parece que se puede ratificar la idea enunciada en un principio: la exploración de las relaciones entre los dos problemas aquí tratados ayuda al examen de ambos. A continuación consideraré el modo en que esto puede tener lugar sin incurrir en un argumento circular.

El examen de la asimetría de nuestras actitudes hacia el futuro y el pasado nos proporciona un argumento a favor de una concepción de las condiciones para nuestra persistencia de carácter sostenibilista. Hay que indicar que este argumento no es, por supuesto, absolutamente concluyente. De hecho, lo que ocurre es que no parece que haya una solución clara a la cuestión. El motivo de esto radica en que tal solución dependerá del modo en que nos identificamos nosotros mismos con un tipo u otro de cosa, más que de la existencia en sí, como tal, de los tipos que resultan posibles candidatos a tales efectos. La respuesta que demos a este problema reposará así, en último caso, en nuestras intuiciones. Sin embargo, parece que el argumento aquí aportado puede sernos de una gran ayuda para aclarar estas.

Asimismo, una aproximación a la cuestión de nuestra persistencia da argumentos a favor de la tesis que parece que debemos abrazar en lo que atañe al problema de la temporalidad del valor que es la de la neutralidad. Parece que no tenemos razones de ninguna clase para rechazar la neutralidad. Lo único que nos empuja a tal rechazo son nuestras intuiciones acerca de la preeminencia axiológica del futuro. Pero todo lo demás lleva, en principio, a su rechazo. En condiciones normales, esto sería un motivo 
para la adopción de una postura neutral. Si ello no sucede en un caso como éste, únicamente debido a que las intuiciones a las que me acabo de referir (esto es, al prejuicio hacia el futuro que tenemos), son extraordinariamente fuertes. Es muy difícil, si no imposible, librarnos de ellas ${ }^{16}$. Pero no porque éstas se vean reforzadas por alguna razón que las justifique, pues esto no es así.

Cabe apuntar algo más con respecto al hecho de que los vínculos entre las concepciones teóricas apuntadas circulen en ambas direcciones. Alguien podría dudar de si al sostener esto no se está incurriendo en una petición de principio. Pero no es difícil comprobar que no es así, puesto que las premisas que en cada caso se toman para sostener la tesis en cuestión son diferentes. No es que se defienda la neutralidad temporal del valor apelando a una teoría de la persistencia sostenibilista y ésta, a su vez, apelando a la neutralidad temporal como tal. La idea de que nuestra persistencia depende de un soporte, se ve afirmada mediante la apelación a la asimetría temporal de nuestra capacidad para formar representaciones motivacionales. A su vez, la neutralidad es apoyada por la simetría implicada en la persistencia, sea esta entendida conforme al tridimensionalismo o al tetradimensionalismo. Y ni la asimetría de nuestras capacidades de formar representaciones motivadoras depende de la neutralidad ni la simetría en la persistencia del sostenibilismo, a pesar de que en ambos casos se trata de tesis relacionadas que surgen al tratar un mismo tema.

Todas estas ideas no constituyen, ciertamente una tesis fuerte, más aun, los argumentos presentados para defenderla son ciertamente de carácter muy general, y sin duda serán necesarios desarrollos mayores, más detallados, para examinarlos en toda su amplitud. Con todo, parecen abrir una línea de indagación que puede ser muy fructífera. La intención de este artículo ha sido la de mostrar la fecundidad de una investigación de este tipo, que contraste los resultados que podemos obtener mediante el análisis de dos problemáticas en principio diversas como las aquí consideradas.

\section{Referencias bibliográficas}

Cicerón, M. T. (2005), Conversaciones en Túsculo. Madrid: Asociación Española de Neuropsiquiatría.

Chisholm, R. (1990), "Referring to Things that No Longer Exist", Philosophical Perspectives. 4: 545-556.

Geach, P. T. (1972), Logic Matters. Oxford: Basil Blackwell.

Hume, D. (1978), “A Treatise on Human Nature”. Oxford: Oxford University Press.

Kamm, F. M. (1993), Morality, Mortality. Vol I: Death and Whom to Save From It. Oxford: Oxford University Press.

16 Tras su presentación por Parfit (1984), este dilema ha sido explorado en particular por Persson (2005). 
Locke, J. (1968), An Essay concerning Human Understanding. London: Dent.

Lucrecio, T. (1976), De la naturaleza. Barcelona: Bosch.

McMahan, J. (2002), The Ethics of Killing: Problems at the Margins of Life. Oxford: Oxford University Press.

Parfit, D. (1984), Reasons and Persons. Oxford: Oxford University Press.

Persson, I. (2005), The Retreat of Reason: A Dilemma in the Philosophy of Life. Oxford: Oxford University Press.

Prior, A. N. (1959), “Thanks Goodness that's Over”, Philosophy. 34: 12-17.

Sider, T. (2001), Four-Dimensionalism: An Ontology of Persistence and Time. Oxford: Oxford University Press.

Sidgwick, H. (1996), The Methods of Ethics, $7^{\mathrm{a}} \mathrm{ed}$. Bristol: Thoemmes Press.

Slote, M. (1983), Goods and Virtues. Oxford: Oxford University Press.

Tooley, M. (1997), Time, Tense, and Causation. Oxford: Oxford University Press.

van Inwagen, P. (1990), "Four-Dimensional Objects”, Nô̂s. 24: 245-255.

Wiggins, D. (2001), Sameness and Substance Renewed. Cambridge: Cambridge University Press.

Williams, B. (1970), “The Self and the Future", The Philosophical Review. 79: 161180. 